\title{
Giuseppe Galli (2017): Der Mensch als Mit-Mensch. Aufsätze zur Gestalttheorie in Forschung, Anwendung und Dialog - herausgege- ben und eingeleitet von Gerhard Stemberger. Wien: Verlag Wolfgang Krammer, 208 Seiten, $€$ 25.00, ISBN13: 97-3-901811-75-3.
}

In seinem letzten Lebensjahr arbeitete der italienische Mediziner, Philosoph und Psychologe Giuseppe Galli (1933-2016) gemeinsam mit Gerhard Stemberger an der Herausgabe von Schriften zu Grundkonzepten der Gestalttheorie, zur Theorie und Praxis der Gestaltpsychologie sowie zu zahlreichen Themen der Psychologie und ihrer Nachbarwissenschaften, die unlängst im Wolfgang Krammer Verlag in deutscher Sprache erschienen sind.

Giuseppe Galli war Schüler von Renzo Canestrari an der Universität Bologna, wo er Wolfgang Metzger kennen lernte und bei dessen Vorlesungen in den Zentren der italienischen Gestalttheorie für ihn übersetzte. Er wurde selbst ein bedeutender Gestalttheoretiker und widmete sich in seinem Forschen Fragen zu Ausdruck und Wahrnehmung, zur Psychologie des phänomenalen Ich, zur Psychologie sozialer Tugenden wie zu psychologischen Aspekten von Sprache und Semiotik. Darüber hinaus initiierte er eine Reihe von bedeutsamen Publikations- und Netzwerkprojekten weit über den italienischen Sprachraum hinaus.

Der Inhalt der vorliegenden Aufsatzsammlung zeugt von Gallis weit gefächertem Interesse. Nach einer Einleitung von Gerhard Stemberger sind in den sieben Kapiteln des Buches insgesamt vierundzwanzig kurze und längere Beiträge zusammengestellt, von denen das erste Schriften „zu den Grundkonzepten der Gestalttheorie“ sammelt, im Weiteren geht es um „Die Person im Gleichgewicht von Ich und Wir", „Gestalttheorie der sozialen Tugenden“, „Gestaltpsychologie und Ethik“" „Gestalttheorie in Klinischer Psychologie und Psychotherapie“, „Gestaltpsychologie und Entwicklungspsychologie“ und schließlich um „Gestaltpsychologie und Neurowissenschaften".

Mitmenschlichkeit und Respekt waren für Galli nicht nur eine persönliche Lebensmaxime, sondern bildeten ein mehrdimensionales Prinzip wissenschaftlicher Arbeit (in Erkenntnistheorie, Forschungspraxis, Erziehungsstil). Für Galli erschließt sich die psychische Realität in ihrer phänomenalen Vielschichtigkeit überhaupt erst durch respektvolles Herangehen. Galli weist darauf hin, dass die Entdeckung der Bedeutsamkeit mit-menschlicher (Inter-) Aktion als Methode einen selten beachteten Beitrag der Gestalttheorie zum psychologisches Forschen 
darstellt und verortet dies besonders in der experimentellen Handlungsforschung Lewins und seiner Schüler(innen) sowie in der Auseinandersetzung Wolfgang Metzgers mit der Tiefenpsychologie. Ganzheitliches Vorgehen ist in diesem Sinne nicht unbestimmt oder uferlos, sondern vorsichtig in die Tiefe des Erfahrungsraums dringende „Strukturanalyse“.

Die Betonung des Menschlichen stellt für die Gestalttheoretiker und Galli nicht das „Ich“ in den Mittelpunkt der phänomenalen Welt. Wie schon Wertheimer gezeigt hatte, zentriert sich die phänomenale Welt nur in Ausnahmefällen um ein Ich als Wirkungszentrum, sie strukturiert sich vielmehr in aller Regel um das strukturell Geforderte und umschließt darin Ich, Objekte und wünschbare oder zu vermeidende Wirkungsrichtungen im Sinne einer aktuellen Gesamtdynamik (S. 45f.). Das wird für Galli anschaulich erfahrbar in Werken der Kunst, in denen sich die sichtbare Dynamik oftmals nicht zwischen bestimmten Personen und ihren Objekten entfaltet, sondern gleichsam quer durch die dargestellten Personen und Gegenstände im Sinne einer bildbeherrschenden Figuration.

Die Dezentrierung der Ich-Perspektive führt Galli zu seinem Lieblingsthema, den sozialen Tugenden, die ihn auch zu seinen weit über Italien bekannt gewordenen interdisziplinären Colloquien über Vergebung, Dankbarkeit, Staunen, Hingabe, Vertrauen, Aufrichtigkeit an der Universität Macerata inspirierten. Aus Gallis Sicht sind die Tugenden nicht etwa individuelle Eigenschaften, sondern Kennzeichen einer gelingenden Auflösung von Ichhaftigkeit (Egozentrismus) im Handlungsraum. In Anlehnung an Paul Ricoeur beschreibt er sie als bipolare Tendenzen mit förderlichen und hemmenden Anteilen - dabei ordnet er (strenge) Systemastik gerne dem Zitieren eines unerschöpflichen Vorrats von biografischen Notizen und Begebenheiten aus dem Leben von Zeitzeugen und historischen Persönlichkeiten unter, in denen sich Tugenden konkret manifestieren. So findet sich beispielsweise die bei Ricoeur im Raum zwischen Großherzigkeit und Verpflichtung angesiedelte „Dankbarkeit“ in Goethes Reflexionen zur künstlerischen Begabung genauso wie in Camus Äußerungen über die frühe Förderung seines Talentes durch seinen Lehrer wie in Gallis eigener Einstellung gegenüber der Pflegerin seiner kranken Mutter (S. 69ff.).

Die hier zum Ausdruck kommende Grundtendenz, Erleben und Verhalten in seinen (positiv!) konstituierenden Zügen zu würdigen, bewegt Galli zu einer kritischen Diskussion der Psychoanalyse, die er, in vielem - der phänomenologischen Einstellung und der ganzheitlichen Behandlung ihres Gegenstandes - der Gestalttheorie ähnlich, durch ein eher pessimistisches Menschenbild von dieser unterscheidet. Insbesondere wenn Triebe, Konflikte, Störungen wie Wesenheiten behandelt werden, sieht Galli im gestalttheoretischen Ausgehen von der sich 
im Feld bildenden wirksamen aktuellen Gesamtsituation eindeutige Vorzüge. Umgekehrt hält er der Tiefenpsychologie aber zugute, dass sie die Gestalttheorie - ganz im Sinne von Lewin - dazu herausfordert, die traditionelle Zentrierung auf die „phänomenale Objektivität" des Wahrnehmungsgeschehens im Sinne der „phänomenalen Subjektivität“ von Selbst- und Fremderfahrung zu überwinden und den Lebensraum (Person und Umgebung) als Ganzes zu sehen (S. 103).

Die Vertreter der Gestalttheorie lernten ihren Wirkungsbereich von einer Forschungshaltung im Hinblick auf Entwicklung und Behandlung zu erweitern, als sie sich mit interpersonalen, diskursiven und transitorischen Aspekten auseinandersetzten. Das ist Giuseppe Galli besonders wichtig, und sicher ging Wolfgang Metzger hier voraus. Doch hätte sich aus meiner Sicht auch eine Beschäftigung mit einem Grenzgänger von Psychoanalyse und Gestalttheorie nahegelegt, für den „Übertragung“ als bipolares Feldphänomen zur Grundlage tiefenpsychologischer Arbeit geworden ist, den man aber bislang ausschließlich mit (Ethno-) Psychoanalyse zusammenbringt, Georges Devereux.

Wie ein roter Faden ziehen sich insbesondere sprachliche Aspekte durch Gallis Beobachtungen zu (Gestalt-) Psychologie, Kunsttheorie, Ethik, Neurowissenschaft. Einige der gesammelten Aufsätze konzentrieren sich auf die Terminologie der Gestalttheorie („Bezugssystem“, „Zentrierung“), andere auf komplexe intersemiotische Zusammenhänge wie denjenigen von Text und Bild, wieder andere problematisieren das in ihrer Wissenschaftssprache zum Ausdruck kommende Menschenbild der Psychologie. Immer wieder entdeckt man Gallis Vorliebe für (sprachlich gelungene) Beschreibungen und Reflexionen in der Literatur. Gelegentlich wiederholt sich ein Gedanke oder Zitat im einen oder anderen Zusammenhang.

Die Liebe zur Sprache zeigt sich auch in einem entwicklungspsychologischen Beitrag, in dem er Individuationsprozesse auf gestaltpsychologischer Grundlage beschreibt und dafür ausführlich aus einer autobiografischen Erzählung von Camus, den Jugenderinnerungen eines Kollegen und den Tagebüchern von Angelo Roncalli (dem späteren Papst Johannes XXIII.) zitiert (S. 127ff.). Im gleichen Kapitel findet sich ein Gastbeitrag von Anna Arfelli Galli zum Verständnis frühkindlicher Leistungen; zu Gast ist an anderer Stelle auch Giancarlo Trombini, mit dem Galli einen interessanten Aufsatz zu Bezugssystemen verfasst hat.

Gallis Schriftensammlung ist fundiert, gedankenreich, heterogen, episodisch und kurzweilig. Entsprechend seiner breit gefächerten wissenschaftlichen Herkunft verwebt Galli Grundannahmen der Gestalttheoretiker mit philosophischen Aperçus, biografischen Selbstzeugnissen von Dichtern, Philosophen, Politikern aller Jahrhunderte und einem (meist) impliziten philanthropischen Impetus. 
Es ist Gerhard Stemberger - so kurz nach dem Tode Gallis und ohne eine Endredaktion letzter Hand - vermutlich nicht leicht gefallen, den breit aufgestellten Aufsatzband fertigzustellen. Doch ist er die Aufgabe behutsam und vorsichtig kommentierend angegangen und eröffnet einem deutschen Publikum damit nicht nur den Gedankenreichtum eines bedeutenden Gestalttheoretikers, sondern übergibt den Leserinnen und Lesern zum richtigen Zeitpunkt das wissenschaftliche Testament eines Autors, der nicht durch glanzvolle Systeme und große Gesten in Erinnerung bleiben wird, sondern durch sein sympathisches und zurückgenommenes Agieren mit den Menschen und für die Menschen.

\section{Herbert Fitzek, Berlin}

Herbert Fitzek, geb. 1957, Dipl. Psych., Dr. phil. Habil., psychologischer Psychotherapeut, studierte Psychologie an der Universität zu Köln, wo er als wissenschaftlicher Mitarbeiter am Psychologischen Institut forschte. 2006 Professor für Wirtschafts- und Kulturpsychologie an der Fachhochschule für Management und Kommunikation Potsdam, seit 2010 Prorektor Forschung an der BSP - Business School Berlin. Forschungschwerpunkte in Kulturpsychologie, Organisationspsychologie, Geschichte der Psychologie, Gestaltpsychologie, qualitative Methoden, Kunst Coaching.

Addresse: BSP Business School Berlin, Villa Siemens, Calandrellistraße 1-9, 12247 Berlin, Germany

E-mail: herbert.fitzek@businessschool-berlin.de 